\title{
THOM POLYNOMIALS FOR OPEN WHITNEY UMBRELLAS OF ISOTROPIC MAPPINGS
}

\author{
TORU OHMOTO \\ Department of Mathematics, Kagoshima University \\ Kagoshima, 890 Japan \\ E-mail: ohmoto@sci.kagoshima-u.ac.jp
}

\begin{abstract}
A smooth mapping $f: L^{n} \rightarrow\left(M^{2 n}, \omega\right)$ of a smooth $n$-dimensional manifold $L$ into a smooth $2 n$-dimensional symplectic manifold $(M, \omega)$ is called isotropic if $f^{*} \omega$ vanishes. In the last ten years, the local theory of singularities of isotropic mappings has been rapidly developed by Arnol'd, Givental' and several authors, while it seems that the global theory of their singularities has not been well studied except for the work of Givental' [G1] in the case of dimension 2 (cf. $[\mathrm{A}],[\mathrm{Au}],[\mathrm{I} 2],[\mathrm{I}-\mathrm{O}]$ ). In the present paper, we are concerned with typical singularities with corank 1 of isotropic maps $f: L^{n} \rightarrow\left(M^{2 n}, \omega\right)$ (arbitrary dimension $n$ ), so-called open Whitney umbrellas of higher order, investigated by Givental' [G2], Ishikawa [I1] and Zakalyukin $[\mathrm{Z}]$, and our purpose is to give their topological invariants from the viewpoint of "Thom polynomial theory" (cf. [T], [P], [K], [AVGL]). These are obtained as a variant of Porteous' formulae on Thom polynomials for $A_{k}$-singularities $[\mathrm{P}]$. Throughout this paper, manifolds are assumed to be paracompact Hausdorff spaces and of class $C^{\infty}$, and maps are also of class $C^{\infty}$.
\end{abstract}

1. Introduction. To begin with, we describe local models of singularities of isotropic map-germs, according to Ishikawa [I1]. Consider $\mathbb{R}^{n}$ with coordinates $x_{1}, \ldots, x_{n}$ and $\mathbb{R}^{2 n}$ $\left(=\mathbb{C}^{n}\right)$ with canonical coordinates $p_{1}, \ldots, p_{n} ; q_{1}, \ldots, q_{n}$ defining the standard symplectic structure as $\omega_{0}=\sum_{i=1}^{n} d p_{i} \wedge d q_{i}$. For $1 \leq k \leq\left[\frac{n}{2}\right]$, a map-germ $f_{n, k}: \mathbb{R}^{n}, 0 \rightarrow \mathbb{R}^{2 n}, 0$ is defined by

$$
\begin{gathered}
q_{i} \circ f_{n, k}=x_{i} \quad(1 \leq i \leq n-1), \\
u=q_{n} \circ f_{n, k}=\frac{x_{n}^{k+1}}{(k+1) !}+\sum_{i=1}^{k-1} x_{i} \frac{x_{n}^{k-i}}{(k-i) !}, \\
v=p_{n} \circ f_{n, k}=\sum_{i=0}^{k-1} x_{k+i} \frac{x_{n}^{k-i}}{(k-i) !}
\end{gathered}
$$

1991 Mathematics Subject Classification: 58C27, 58F05.

The paper is in final form and no version of it will be published elsewhere. 


$$
p_{j} \circ f_{n, k}=\int_{0}^{x_{n}}\left(\frac{\partial v}{\partial x_{j}} \frac{\partial u}{\partial x_{n}}-\frac{\partial v}{\partial x_{n}} \frac{\partial u}{\partial x_{j}}\right) d x_{n} \quad(1 \leq j \leq n-1) .
$$

Each $f_{n, k}$ is isotropic with respect to $\omega_{0}$, and has corank 1 (kernel rank 1 ) singularities. We remark that $f_{n, k}$ can be considered as an "isotropic lifting" of $\left(q_{1}, \ldots, q_{n}, p_{n}\right) \circ f_{n, k}$ : $\mathbb{R}^{n} \rightarrow \mathbb{R}^{n+1}$, which is the normal form of Morin singularity of order $k$.

Let $L$ be an $n$-manifold, $(M, \omega)$ a symplectic $2 n$-manifold and $f: L \rightarrow M$ an isotropic mapping. Throughout this paper, we say that the germ of $f: L, p \rightarrow M, f(p)$ at $p$ is of open Whitney umbrella type of order $k$ if it is transformed to the normal form $f_{n, k}$ by means of suitable choices of local coordinates of $L$ centered $p$ and a symplectic local coordinate of $M$ centered at $f(p)$.

We intend to represent singularities of $f$ of open Whitney umbrella type in a geometrical way, as Thom-Boardman singularities $\Sigma_{1^{k}}(f)\left(=\Sigma_{1, \ldots, 1}(f)\right.$ with 1 repeated $k$ times). The intuitive meaning of this notation is as follows. First, set $\Sigma_{1}(f)$ to be $\left\{x \in L \mid \operatorname{dim} \operatorname{ker} d f_{x}=1\right\}$. If $\Sigma_{1}(f)$ becomes a $C^{\infty}$ submanifold of $L$, then considering the restriction of $f$ to $\Sigma_{1}(f)$, which is also an isotropic mapping into $M$, set again $\Sigma_{1,1}(f):=\Sigma_{1}\left(\left.f\right|_{\Sigma_{1}(f)}\right)$. Inductively, we define $\Sigma_{1^{k}}(f)$ by $\Sigma_{1}\left(\left.f\right|_{\Sigma_{1 k-1}(f)}\right)$ as far as possible, and then we obtain a sequence of submanifolds: $L \supset \Sigma_{1}(f) \supset \Sigma_{1,1}(f), \ldots$ In $\S 2$ and $\S 3$ we will make this procedure rigorous in terms of intrinsic derivatives associated to $f$ and certain transversality conditions, so that the germ of $f$ at each point of $\Sigma_{1^{k}}(f)-\Sigma_{1^{(k+1)}}(f)$ is of open Whitney umbrella type of order $k$. Then, it turns out that $\Sigma_{1^{k}}(f)$ becomes a closed submanifold in $L$ with codimension $2 k$, which is canonically co-oriented (the normal bundle is oriented). Hence, in particular, for oriented $L$, it defines $(n-2 k)$-dimensional homology class of $L$ with $\mathbb{Z}$-coefficients.

Recall that every symplectic manifold $(M, \omega)$ admits an almost complex structure $J$, unique up to homotopy, such that $\omega(*, J *)$ is positive definite (see [W]). In particular, we can define the Chern classes $c_{i}(T M) \in H^{2 i}(M ; \mathbb{Z})$, which depend only on the symplectic structure.

We now state our results. Let $L$ be always assumed to be compact without boundary.

TheOREM (1.1). Let $f: L^{n} \rightarrow M^{2 n}$ be an isotropic map whose differential $d f_{x}$ at each $x \in L$ has corank at most 1 . Assume $L$ is oriented. Then

$$
2 c_{i}\left(f^{*} T M-T L_{\mathbb{C}}\right)=0 \quad \text { for all } i \geq 2 .
$$

(Here $T L_{\mathbb{C}}$ denotes the complexification of the tangent bundle $T L$, and the Chern class $c_{i}\left(f^{*} T M-T L_{\mathbb{C}}\right) \in H^{2 i}(L ; \mathbb{Z})$ is the $i$-th term of $c\left(f^{*} T M\right) \bar{c}\left(T L_{\mathbb{C}}\right)$. $)$

We shall express the cohomology classes dual to $\Sigma_{1^{k}}(f)$ by some polynomials of the standard characteristic classes of the bundles $T L$ and $f^{*} T M$, that may be called Thom polynomials for higher open Whitney umbrellas. These provide characteristic classes for isotropic mappings, which constitute topological obstructions to the existence of the corresponding open umbrellas.

Theorem (1.2). Let $f: L^{n} \rightarrow M^{2 n}$ be an isotropic map whose singularities are only of open Whitney umbrella type, $\Sigma_{1^{k}}(f)\left(1 \leq k \leq\left[\frac{n}{2}\right]\right)$. Then: 
(1) if $L$ is oriented, the Poincaré dual to $\Sigma_{1^{k}}(f)$ is $\left[\Sigma_{1^{k}}(f)\right]^{c}=f^{*} c_{1}(T M)^{k}$ modulo 2-torsion elements for each $k$;

(2) if $L$ is unoriented, taking (co)homology with $\mathbb{Z}_{2}$-coefficients, the dual to $\Sigma_{1^{k}}(f)$ is $\left[\Sigma_{1^{k}}(f)\right]_{2}^{c}=\sum_{i=1}^{k}\left(\begin{array}{c}{[k / 2]} \\ i-1\end{array}\right) w_{2}^{k-i} w_{2 i}$ where $w_{i}$ means the $i$-th Stiefel-Whitney class $w_{i}\left(f^{*} T M\right.$ $-T L \oplus T L)$.

Theorem (1.1) implies a restriction on the Pontryagin classes of $L$. For example, it is straightforward that

Corollary (1.3). If $L$ has some non-trivial rational Pontrjagin classes, then any proper isotropic mapping $g$ from $L$ into $\mathbb{R}^{2 n}$ (furnished with any symplectic structure) has singularities of corank greater than 1 , i.e., $\Sigma_{r}(g)\left(=\left\{x \in L \mid \operatorname{dim} \operatorname{ker} d g_{x}=r\right\}\right) \neq \emptyset$ for some $r \geq 2$.

We can also see that the dual classes to open umbrellas give homotopy invariants of the isotropic map $f$ :

Corollary (1.4). Let $f$ and $g$ be two isotropic maps $L^{n} \rightarrow\left(M^{2 n}, \omega\right)$ which have only singularities of open Whitney umbrella type. If $f$ is continuously homotopic to $g$, then their singularity sets of order $k$ define the same homology class of $L$ for each $k$ (i.e. $\left[\Sigma_{1^{k}}(f)\right]=\left[\Sigma_{1^{k}}(g)\right] \in H_{n-2 k}(L ; \mathbb{Z})$ for oriented $\left.L\right)$.

Note that to more degenerate rank singularities of isotropic maps, $\Sigma_{r}(f)(r \geq 2)$, we cannot apply our approach because of the lack of transversality theorems, see $\S 2$. Recall that we are far from a classification of generic isotropic map-germs with corank greater than 1 , see $[\mathrm{A}]$.

In the case of dimension 2, we can derive from our theorems some integral formula of "local Maslov indices" first introduced by Givental' (see also [I-O], [I2]). Consider an isotropic mapping $f$ from a closed surface $S$ to a symplectic 4 -manifold $(M, \omega)$ such that singular points of $f$ are all isolated (not necessarily of corank 1). For each point $p$ of $S$, we can define the index $m(f, p) \in \mathbb{Z}$ (resp. $\left.m(f, p)_{2} \in \mathbb{Z}_{2}\right)$ if $L$ is oriented (resp. unoriented), see $\S 5$. We can see that summing $m(f, p)$ over all points of $L$ yields some characteristic numbers.

Corollary (1.5). Let $f$ be an isotropic mapping from a closed surface $S$ to a 4dimensional symplectic manifold $(M, \omega)$. Assume that the singularities of $f$ are all isolated. Then:

$$
\begin{gathered}
\sum_{p \in S} m(f, p)=c \quad \text { if } S \text { is oriented, } \\
\sum_{p \in S} m(f, p)_{2} \equiv \chi+w(\bmod 2) \quad \text { if } S \text { is unoriented. }
\end{gathered}
$$

Here $\chi$ denotes the mod 2 Euler number of $S$, and $c$ (resp. $w)$ denotes the characteristic number $\left\langle f^{*} c_{1}(T M),[S]\right\rangle\left(\operatorname{resp} .\left\langle f^{*} w_{2}(T M),[S]_{2}\right\rangle\right)$.

R e m ark (1.6). We note that $m(f, p)=0$ for non-singular points $p$ of $f$, hence the left hand sides of the above formulae make sense. In particular, if the germ of $f$ at $p$ is of open Whitney umbrella type of order 1 , then $m(f, p)= \pm 1$. If the tangent bundle $T M^{4}$ 
admits some Lagrangian subbundle, then the Chern number $c$ vanishes, hence the sum of Maslov indices is zero. This fact has been indicated by Givental' ([G1]). We should remark that in the same paper, Givental' proved a strong formula on the number of open umbrellas and of transversal double points, with which we do not deal here. A generalization of the formulae is given in [I-O].

Acknowledgements. The author wish to express his gratitude to Professor G. Ishikawa for his help and interest in this paper motivated by his papers [I1], [I2] and Givental's [G1], and also to Professor S. Izumiya for his encouragement.

2. Intrinsic derivatives of isotropic bundle maps. Let $W$ be a real vector space with a symplectic structure $\omega$ (i.e. non-degenerate skewsymmetric bilinear form). For a subspace $I$ of $W$, the $\omega$-orthogonal space $I^{\perp}$ is defined to be the set of $w$ in $W$ such that $\omega(w, v)=0$ for any $v \in I$, and we always have $\operatorname{dim} I+\operatorname{dim} I^{\perp}=\operatorname{dim} W$. A subspace $I$ is called isotropic if $I \subset I^{\perp}$. We remark that for isotropic $I$, the quotient $I^{\perp} / I$ has the natural symplectic structure $\omega_{I^{\perp}}$ defined by $p^{*} \omega_{I^{\perp}}=\left.\omega\right|_{I^{\perp}}$ where $p$ is the projection $I^{\perp} \rightarrow I^{\perp} / I$.

A linear map from a real vector space $V$ to $W$ is also called isotropic if its image is an isotropic subspace of $W$, and let $I L(V, W)$ denote the set of all isotropic linear maps from $V$ to $W$. Then $I L(V, W)$ becomes a real homogeneous subvariety of the space $\operatorname{Hom}_{\mathbb{R}}(V, W)$, since it is defined by quadratic equations $h^{*} \omega=0$, where $h \in \operatorname{Hom}_{\mathbb{R}}(V, W)$. In particular, $I L(V, W)$ coincides with $\operatorname{Hom}_{\mathbb{R}}(V, W)$ if and only if $\operatorname{dim} V=1$.

Let $\Sigma_{r}(V, W)$ denote the submanifold consisting of linear maps $V \rightarrow W$ with corank $r$, and set $I \Sigma_{r}(V, W):=\Sigma_{r}(V, W) \cap I L(V, W)$. Then

$$
I L(V, W)=\bigcup_{r \geq 0} I \Sigma_{r}(V, W), \quad \overline{I \Sigma_{s}(V, W)}=\bigcup_{r \geq s} I \Sigma_{r}(V, W) .
$$

Here bar means closure in $\operatorname{Hom}_{\mathbb{R}}(V, W)$. Clearly, $I \Sigma_{r}(V, W)$ is a semi-algebraic subset, which is an orbit of the canonical right-left actions of the general linear transformations on $V$ and the symplectic linear transformations on $W$. Let $h$ be a point of $I \Sigma_{r}(V, W)$. To see the local geometric structure of $I L(V, W)$ around $h$, it is useful to define a kind of "tangent cone" of $I L(V, W)$ at $h$ : let Cone $r(h)$ denote the set of velocity vectors $\frac{d c}{d s}(0) \in T\left(\operatorname{Hom}_{\mathbb{R}}(V, W)\right)_{h}$ for all smooth curve-germs $c:(\mathbb{R}, 0) \rightarrow(I L(V, W), h)$.

It is well-known (see e.g. $[\mathrm{P}],[\mathrm{G}-\mathrm{G}])$ that over $\Sigma_{r}(V, W)$ the kernel bundle $K$, the image bundle $I$ and the cokernel bundle $C$ are canonically defined, and there exists an exact sequence of bundle morphisms

$$
0 \rightarrow T \Sigma_{r}(V, W) \rightarrow T\left(\operatorname{Hom}_{\mathbb{R}}(V, W)\right) \stackrel{\pi}{\rightarrow} \operatorname{Hom}_{\mathbb{R}}(K, C) \rightarrow 0 .
$$

The projection $\pi$ is given by assigning to each vector $\alpha$ the composite linear map $K_{h} \hookrightarrow V \stackrel{\alpha}{\rightarrow} W \rightarrow C_{h}$ through the natural identification between $T\left(\operatorname{Hom}_{\mathbb{R}}(V, W)\right)_{h}$ and $\operatorname{Hom}_{\mathbb{R}}(V, W)$.

Proposition (2.1). (1) For any $h \in I \Sigma_{r}(V, W), \pi$ Cone $\left._{r}(h)\right)=I L\left(K_{h},\left(I^{\perp} / I\right)_{h}\right)$ where $K_{h}=\operatorname{ker} h$ and $I_{h}=$ image $h$. 
(2) The singular locus of $I L(V, W)$ coincides with $\overline{I \Sigma_{2}(V, W)}$, while $I \Sigma_{1}(V, W)$ is a smooth submanifold of the regular locus of $I L(V, W)$ whose normal bundle is isomorphic to $\operatorname{Hom}_{\mathbb{R}}\left(K,\left(I^{\perp} / I\right)\right)\left(=I L\left(K,\left(I^{\perp} / I\right)\right)\right)$.

Proof. (1) We show $\pi\left(\right.$ Cone $\left._{r}(h)\right) \subset I L\left(K_{h},\left(I^{\perp} / I\right)_{h}\right)$. For any $\alpha \in$ Cone $_{r}(h)$, choose a smooth curve $c(s)$ in $I L(V, W)$ such that $c(0)=h$ and $\frac{d c}{d s}(0)=\alpha$. Also set $\beta=\frac{d^{2} c}{d s^{2}}(0)$, and consider $\alpha$ and $\beta$ as elements of the linear space $\operatorname{Hom}_{\mathbb{R}}(V, W)$. Since $\omega(c(s) u, c(s) v)$ is constantly zero for $u, v \in V$, taking derivatives at $s=0$ gives $\omega(\alpha u, h v)+\omega(h u, \alpha v)=0$ and $2 \omega(\alpha u, \alpha v)+\omega(\beta u, h v)+\omega(h u, \beta v)=0$. It follows that $\omega(\alpha u, h v)=0$ for $u \in K_{h}, v \in$ $V$, and that $\omega(\alpha u, \alpha v)=0$ for $u, v \in K_{h}$. The former implies that $\alpha\left(K_{p}\right) \subset I_{p}{ }^{\perp}$, and hence $\pi(\xi)$ is defined as a linear map $K_{h} \rightarrow I_{h}^{\perp} / I_{h}$. By the latter, we see $\pi(\xi)$ is isotropic with repect to $\omega_{I_{h}}$. To see the converse inclusion is easy.

(2) Define $\tau: \operatorname{Hom}_{\mathbb{R}}(V, W) \rightarrow V^{*} \wedge V^{*}$ by $\tau(h):=h^{*} \omega$. Then $\tau^{-1}(0)=I L(V, W)$. For $h \in I L(V, W)$, it can be shown that the differential $d \tau$ at $h$ is surjective if and only if $h$ has corank at most 1 , see [I2]. Hence any points of strata $I \Sigma_{0}$ and $I \Sigma_{1}$ are regular points of $I L(V, W)$. If the corank of $h$ is greater than $1, I L\left(K_{h},\left(I^{\perp} / I\right)_{h}\right)$ is not a linear space, hence Cone $_{r}(h)$ is also not linear. Thus each point of $\overline{I \Sigma_{2}(V, W)}$ is a singular point of $I L(V, W)$. Thus the assertion (2) follows.

Next, we shall work in the category of $C^{\infty}$ vector bundles. Assume that we are given a real vector bundle $E \rightarrow X$ over a manifold $X$, and a vector bundle $F \rightarrow X$ with a smooth symplectic structure $\omega$, that is, a smooth section of $F \wedge F$ which is non-degenerate at each $x \in X$. Let $I L(E, F)$ be the union of $I L\left(E_{x}, F_{x}\right)$ over all $x \in X$. We call a smooth section of $I L(E, F)$ an isotropic bundle map.

Let $h: E \rightarrow F$ be a bundle map. We denote by $\Sigma(h)$ the set of points in $X$ at which $h$ is not injective. Let $p$ be a point of $\Sigma(h)$, and recall the intrinsic derivative $(D h)_{p}$ of $h$ at $p$ (e.g. [G-G]) which is given by the composition

$$
T X_{p} \rightarrow T\left(\operatorname{Hom}_{\mathbb{R}}(E, F)\right)_{h(p)} \stackrel{\pi}{\rightarrow} \operatorname{Hom}_{\mathbb{R}}\left(\operatorname{ker} h_{p}, \operatorname{coker} h_{p}\right) .
$$

When $h$ is an isotropic bundle map, from (1) of Proposition (2.1) we easily have the following restriction of the derivative. For simplicity, set $K_{p}=\operatorname{ker} h_{p}, I_{p}=$ image $h_{p}$ and $C_{p}=$ coker $h_{p}\left(=F_{p} / I_{p}\right)$.

Proposition (2.2). An isotropic bundle map $h: E \rightarrow F$ induces the canonically defined intrinsic derivative

$$
(D h)_{p}: T X_{p} \rightarrow \operatorname{Hom}_{\mathbb{R}}\left(K_{p},\left(I^{\perp} / I\right)_{p}\right)
$$

such that the image $(D h)_{p}\left(T X_{p}\right)$ is contained in $I L\left(K_{p},\left(I^{\perp} / I\right)_{p}\right)$.

Let $I \Sigma_{1}(E, F)$ denote the union of $I \Sigma_{1}\left(E_{x}, F_{x}\right)$ over all $x \in X$. Propositions $(2.1)$ and (2.2) yield

Proposition (2.3). Let $h: E \rightarrow F$ be an isotropic bundle map, and $p$ a singular point of $h$ with $\operatorname{dim}_{\mathbb{R}} K_{p}=1$. Then the following properties are equivalent:

(1) $(D h)_{p}: T X_{p} \rightarrow \operatorname{Hom}_{\mathbb{R}}\left(K_{p},\left(I^{\perp} / I\right)_{p}\right)\left(=I L\left(K_{p},\left(I^{\perp} / I\right)_{p}\right)\right)$ is surjective,

(2) the section $h: X \rightarrow I L(E, F)$ is transverse to $I \Sigma_{1}(E, F)$ at $p$. 
Remark (2.4). (1) By standard arguments on transversality, we can see that any isotropic bundle map $E \rightarrow F$ with corank at most 1 can be approximated by isotropic bundle maps transverse to $I \Sigma_{1}(E, F)$.

(2) If $\operatorname{dim}_{\mathbb{R}} K_{p}$ is greater than 1 , then $(D h)_{p}: T X_{p} \rightarrow \operatorname{Hom}_{\mathbb{R}}\left(K_{p},\left(I^{\perp} / I\right)_{p}\right)$ is never surjective.

3. Higher order singularities. For an isotropic mapping $f: L^{n} \rightarrow M^{2 n}$, we now consider the isotropic bundle map $d_{1}:=d f: T L \rightarrow f^{*} T M$ defined over $L$. We simply denote $f^{*} T M$ by $T M^{\prime}$.

Let $\Sigma_{1}(f)$ denote the set of points of $L$ at which $\operatorname{dim}$ ker $d_{1}=1$. Assume that the intrinsic derivative of $d_{1},\left(D d_{1}\right)_{p}: T L_{p} \rightarrow \operatorname{Hom}_{\mathbb{R}}\left(K_{p},\left(I^{\perp} / I\right)_{p}\right)$, is surjective for any $p \in \Sigma_{1}(f)$. Then by Proposition (2.3), $\Sigma_{1}(f)$ is smooth and we have the exact sequence

$$
\left.0 \rightarrow T \Sigma_{1}(f) \rightarrow T L\right|_{\Sigma_{1}(f)} \stackrel{D d_{1}}{\rightarrow} \operatorname{Hom}_{\mathbb{R}}\left(K, I^{\perp} / I\right) \rightarrow 0 \quad \text { over } \Sigma_{1}(f)
$$

Next, we let $d_{2}$ denote the restriction of $D d_{1}$ to $K$,

$$
d_{2}=\left.\left(D d_{1}\right)\right|_{K}: K \rightarrow \operatorname{Hom}_{\mathbb{R}}\left(K, I^{\perp} / I\right) \quad \text { over } \Sigma_{1}(f) .
$$

Considering $d_{2}$ as a section of $\operatorname{Hom}_{\mathbb{R}}\left(K \otimes K, I^{\perp} / I\right)$, let $\Sigma_{1,1}(f)$ be the pull back of the zero section $d_{2}^{-1}(0)$. Clearly, if $d_{2}$ is transversal to the zeros and $d_{2}^{-1}(0)$ is not empty, then $\Sigma_{1,1}(f)$ is smooth and we have

$$
\left.0 \rightarrow T \Sigma_{1,1}(f) \rightarrow T \Sigma_{1}(f)\right|_{\Sigma_{1,1}(f)} \stackrel{D d_{2}}{\longrightarrow} \operatorname{Hom}_{\mathbb{R}}\left(K \otimes K, I^{\perp} / I\right) \rightarrow 0 \quad \text { over } \Sigma_{1,1}(f),
$$

where $D d_{2}$ is the intrinsic derivative of $d_{2}$. Inductively, for $k \geq 2$, we define the $k$ th order intrinsic derivative of $f$ by $d_{k}:=\left.D d_{k-1}\right|_{K}$, which is regarded as a section

$$
d_{k}: \Sigma_{1^{k-1}}(f) \rightarrow \operatorname{Hom}_{\mathbb{R}}\left(\stackrel{k}{\otimes} K, I^{\perp} / I\right),
$$

and define $\Sigma_{1^{k}}(f)$ to be $d_{k}{ }^{-1}(0)$. Also the transversality of $d_{k}$ to the zeros implies that $\Sigma_{1^{k}}(f)$ is an empty set or becomes a submanifold of codimension $2 k$ which is co-orientable via the orientation of $I^{\perp} / I$. In particular, $\Sigma_{1^{k}}(f)=\emptyset$ for $2 k>n$.

It is straightforward from [G2] and [I1] to see the following.

Proposition (3.1). The following properties are equivalent:

(1) the germ of $f$ at $p$ is of open Whitney umbrella type of order $k$,

(2) the intrinsic derivatives $d_{s}(1 \leq s \leq k)$ at $p$ are well-defined (transversal properties mentioned above are all fulfilled), and $d_{k+1}^{-1}(0)=\emptyset$.

Note. Let $p \in L$ be a singular point of an isotropic $f: L \rightarrow(M, \omega)$ with corank 1. Choose a symplectic chart $\left(U,\left(p_{1}, \ldots, p_{n}, q_{1}, \ldots, q_{n}\right)\right)$ of $M$ centered at $f(p)$ such that the image $I_{p}$ of the differential $d f$ at $p$ is spanned by vectors $\frac{\partial}{\partial q_{1}}(0), \ldots, \frac{\partial}{\partial q_{n-1}}(0)$. The $\omega$-orthogonal space $I_{p}^{\perp}$ is spanned by $\frac{\partial}{\partial q_{1}}(0), \ldots, \frac{\partial}{\partial q_{n}}(0)$, and $\frac{\partial}{\partial p_{n}}(0)$. Then our intrinsic derivative $d_{k}$ of the isotropic $f$ at $p$ is the same as the usual $k$ th intrinsic derivative of the map $f^{\prime}:=\left(q_{1}, \ldots, q_{n}, p_{n}\right) \circ f: U \rightarrow \mathbb{R}^{n+1}$ at $p$, therefore (2) in Proposition (3.1) is equivalent to the germ of $f^{\prime}$ at $p$ being of Morin type of order $k$.

As a remark, Ishikawa proved the following genericity theorem (see also [Z]). 
THEOREM (3.2) [I1]. There exists an open and dense subset $\mathcal{O}$ in the space of isotropic mappings $L \rightarrow M$ with corank at most 1 (furnished with Whitney $C^{\infty}$ topology) such that for any element $f \in \mathcal{O}$, and for any $p \in L$, the germ of $f$ at $p$ is of open Whitney umbrella type of order $k$ (for some $0 \leq k \leq[n / 2])$.

4. Proofs of theorems. Before starting the proofs, we state an elementary lemma in linear algebra. Let $V, W$ be vector spaces treated in $\S 2$. We can choose an arbitrary complex structure $J: W \rightarrow W$ compatible with $\omega$, i.e. $J^{2}=-1$ and $\omega(*, J *)>0$. Associated to an $\mathbb{R}$-linear map $l: V \rightarrow W$, we define a $\mathbb{C}$-linear map $\rho(l): V_{\mathbb{C}}(:=$ $\left.V \otimes_{\mathbb{R}} \mathbb{C}\right) \rightarrow W$ by $\rho(l)(u+\sqrt{-1} v):=l(u)+J l(v)$.

Lemma (4.1). If a linear map $l: V \rightarrow W$ is isotropic, then $\operatorname{ker} \rho(l)=(\operatorname{ker} l)_{\mathbb{C}}$ in $V_{\mathbb{C}}$, and $I^{\perp} / I$ (where $I$ denotes image $l$ ) is isomorphic to the underlying real space of $\operatorname{coker} \rho(l)$.

Proof. For isotropic $l$, its image $I$ is totally real with respect to $J$, i.e. $I \cap J I=\{0\}$.

Rem ark (4.2). $\operatorname{Hom}_{\mathbb{R}}(V, W)$ is identified with $\operatorname{Hom}_{\mathbb{C}}\left(V_{\mathbb{C}}, W\right)$ via $\rho$. Let $\Sigma_{r}\left(V_{\mathbb{C}}, W\right)$ be the submanifold which consists of all $\mathbb{C}$-linear maps with complex corank $r$. Then the normal space to $\Sigma_{r}\left(V_{\mathbb{C}}, W\right)$ at an element $\sigma$ is $(\mathbb{C}$ - $)$ isomorphic to $\operatorname{Hom}_{\mathbb{C}}(\operatorname{ker} \sigma, \operatorname{coker} \sigma)$, and in particular, if $\sigma=\rho(l)$ where $l$ is isotropic, by Lemma (4.1) the underlying real space is $\operatorname{Hom}_{\mathbb{R}}\left(K, I^{\perp} / I\right)$. When corank $r=1, I \Sigma_{1}(V, W)$ is the transverse intersection of $\Sigma_{1}\left(V_{\mathbb{C}}, W\right)$ and the regular locus of $I L(V, W)$.

Now let $f: L \rightarrow M$ be an isotropic map whose singularities are only of (generalized) open umbrella type. Fix an almost complex structure $J$ on the bundle $T M^{\prime}$ compatible with $\omega$. Then we can apply in the same way as above the tautological identification $\rho$ to the isotropic bundle map $d_{1}(=d f), d_{2}, \ldots$ described in the previous section, and hence we have smooth complex bundle maps

$$
\begin{aligned}
& \phi_{1}\left(:=\rho\left(d_{1}\right)\right): T L_{\mathbb{C}} \rightarrow T M^{\prime}, \\
& \phi_{k}\left(:=\rho\left(d_{k}\right)\right): K_{\mathbb{C}} \rightarrow \operatorname{Hom}_{\mathbb{C}}\left(\stackrel{k-1}{\otimes} K_{\mathbb{C}}, Q\right) \quad \text { over } \Sigma_{1^{k-1}}(f)(2 \leq k \leq[n / 2]),
\end{aligned}
$$

where $K_{\mathbb{C}}=(\operatorname{ker} d f)_{\mathbb{C}}=\operatorname{ker} \phi_{1}$ and $Q:=$ coker $\phi_{1}\left(\simeq I^{\perp} / I\right)$. It easily follows from Lemma (4.1), Proposition (2.3) and Remark (4.2) that the section $\phi_{1}$ of $\operatorname{Hom}_{\mathbb{C}}\left(T L_{\mathbb{C}}, T M^{\prime}\right)$ is transverse to $\Sigma_{1}\left(T L_{\mathbb{C}}, T M^{\prime}\right)$, and that $\Sigma_{1}(f)$ coincides with the pull buck $\Sigma_{1}\left(\phi_{1}\right)$. For each $2 \leq k, \Sigma_{1^{k}}(f)$ is now represented by $\phi_{k}^{-1}(0)$ as well.

In what follows, we shall apply Porteous' method $([\mathrm{P}])$ to the complex bundle maps $\phi_{k}$ $(k=1,2, \ldots)$ in order to compute the dual classes $\left[\Sigma_{1^{k}}(f)\right]^{c} \in H^{2 k}(L ; \mathbb{Z})\left(\right.$ and $\left[\Sigma_{1^{k}}(f)\right]_{2}^{c} \in$ $\left.H^{2 k}\left(L ; \mathbb{Z}_{2}\right)\right)$. We assume that $L$ is oriented.

Let $P\left(T L_{\mathbb{C}}\right) \stackrel{\pi}{\rightarrow} L$ be the smooth $\mathbb{C} P^{n-1}$-bundle associated to $T L_{\mathbb{C}}$, and $\xi$ the tautological complex line bundle over $P\left(T L_{\mathbb{C}}\right)$. As usual, $\bar{\xi}$ denotes the dual to $\xi$. Now we consider the $\mathbb{C}^{n}$-bundle $\operatorname{Hom}_{\mathbb{C}}\left(\xi, \pi^{*} T M^{\prime}\right)$ over $P\left(T L_{\mathbb{C}}\right)$, and we define a smooth section $\Phi$ of this bundle by $\Phi(x, \lambda)=\left.\left(\phi_{1}\right)_{x}\right|_{\lambda}: \lambda \rightarrow T M_{x}^{\prime}$ where $x \in L$ and $\lambda$ is a complex line in $T L_{x \mathbb{C}}$. Let $Z$ be the pull buck of the zero section via $\Phi, Z:=\Phi^{-1}(0)$. By the same argument as in $\S 1$ of $[\mathrm{P}]$ (and as in Proposition (1.1) of $[\mathrm{R}]$ ), we see that the transversality of $\phi_{1}$ and the submanifold $\Sigma_{1}\left(T L_{\mathbb{C}}, T M^{\prime}\right)$ yields the following properties. 
Proposition (4.3). (1) The section $\Phi$ is transverse to the zero section of the bundle $\operatorname{Hom}_{\mathbb{C}}\left(\xi, \pi^{*} T M^{\prime}\right)$, and hence the pull back $Z$ of zeros becomes a $C^{\infty}$ submanifold of $P\left(T L_{\mathbb{C}}\right)$.

(2) $Z$ is diffeomorphic to $\Sigma_{1}(f)$ via the projection $\left.\pi\right|_{Z}$.

Thus, the Poincaré dual to the homology class $[Z]$ of $H^{2 n}\left(P\left(T L_{\mathbb{C}}\right) ; \mathbb{Z}\right)$, denoted by $[Z]^{c}$, is equal to the top Chern class $c_{n}\left(\bar{\xi} \otimes \pi^{*} T M^{\prime}\right)$ (cf. [MS], [K]). Now we let $\pi_{\text {! denote }}$ the Gysin homomorphism $H^{2(m+n)}\left(P\left(T L_{\mathbb{C}}\right) ; \mathbb{Z}\right) \rightarrow H^{2(m+1)}(L ; \mathbb{Z})$.

Proposition (4.4). We have

$$
\pi_{!}\left(c_{1}(\bar{\xi})^{m} c_{n}\left(\bar{\xi} \otimes \pi^{*} T M^{\prime}\right)\right)=c_{m+1}\left(T M^{\prime}-T L_{\mathbb{C}}\right), \quad m \geq 0 .
$$

It follows from (4.4) that

$$
\left[\Sigma_{1}(f)\right]^{c}\left(=\pi_{!}[Z]^{c}\right)=c_{1}\left(T M^{\prime}-T L_{\mathbb{C}}\right) .
$$

Proof. This proposition follows directly from Porteous' projection formula.

Lemma (4.6) $([\mathrm{P}])$. Suppose $E \stackrel{p}{\rightarrow} X$ is a $\mathbb{C}^{n}$-bundle over an oriented manifold $X$. Let $\nu$ denote the canonical line bundle over $P(E)$. Then $p_{!}\left(c_{1}(\bar{\nu})^{s}\right)=\bar{c}_{s-n+1}(E)$. (Here $\bar{c}_{s-n+1}(E)$ are the normal Chern classes of $E$.)

This is immediately proved from $p_{!}\left(c_{1}(\bar{\nu})^{n-1}\right)=1$ (Proposition 0.3 of $[\mathrm{P}]$ ) and $\sum_{i=0}^{n} p^{*} c_{i}(E) c_{1}(\bar{\nu})^{n-i}=0$. Hence, we have

$$
\begin{aligned}
\pi_{!}\left(c_{1}(\bar{\xi})^{m} c_{n}\left(\bar{\xi} \otimes \pi^{*} T M^{\prime}\right)\right) & =\pi_{!}\left(\sum_{i=0}^{n} \pi^{*} c_{n-i}\left(T M^{\prime}\right) c_{1}(\bar{\xi})^{m+i}\right) \\
& =\sum_{i=0}^{n} c_{n-i}\left(T M^{\prime}\right) \bar{c}_{m+i-n+1}\left(T L_{\mathbb{C}}\right)=c_{m+1}\left(T M^{\prime}-T L_{\mathbb{C}}\right) .
\end{aligned}
$$

Rem ark (4.7). In Lemma (4.6), if $X$ is unoriented, then we consider underlying real bundles of $E$ and $\nu$. By using the Gysin homomorphism between $\mathbb{Z}_{2}$-cohomology groups we have $p_{!}\left(w_{2}(\bar{\nu})^{s}\right)=\bar{w}_{2(s-n+1)}(E)$ (cf. [MS]).

Proof of Theorem (1.1). We define a section $j: \Sigma_{1}(f) \rightarrow P\left(T L_{\mathbb{C}}\right)$ to be the inverse of $\left.\pi\right|_{Z}: Z \rightarrow \Sigma_{1}(f)$. Then $j^{*} \xi=\operatorname{ker} \phi_{1}=K_{\mathbb{C}}$ and $c_{1}(\xi)^{m} j_{!}(1)(m \geq 1)$ are 2-torsion elements of $H^{*}\left(P\left(T L_{\mathbb{C}}\right) ; \mathbb{Z}\right): 2 c_{1}(\xi)^{m} j_{!}(1)=j_{!}\left(2 j^{*} c_{1}(\xi)^{m}\right)=j_{!}\left(2 c_{1}\left(K_{\mathbb{C}}\right)^{m}\right)=0$. Since $j_{!}(1)=[Z]^{c}=c_{n}\left(\bar{\xi} \otimes \pi^{*} T M^{\prime}\right)$, it follows from (4.4) that

$$
2 c_{m+1}\left(T M^{\prime}-T L_{\mathbb{C}}\right)=0 \quad(m \geq 1) .
$$

Considering (1) of Remark (2.4) for $d_{1}$ (or Theorem (3.2)), (4.8) implies the assertion of Theorem (1.1).

Proof of Theorem (1.2). Let us compute explicit forms of $\left[\Sigma_{1^{k}}(f)\right]^{c}$ for $k \geq 2$ in terms of characteristic classes. Let $i_{k}$ denote the inclusion $\Sigma_{1^{k}}(f) \hookrightarrow \Sigma_{1}(f)\left(i_{1}=\mathrm{id}\right)$, and $i^{\prime}$ the inclusion $\Sigma_{1^{k}}(f) \hookrightarrow \Sigma_{1^{k-1}}(f)$.

Let $c_{i}$ stand for $c_{i}\left(T M^{\prime}-T L_{\mathbb{C}}\right)$. Note that $T L_{\mathbb{C}}-K_{\mathbb{C}} \simeq I_{\mathbb{C}} \simeq T M^{\prime}-Q$ over $\Sigma_{1}(f)$. Then, by the definition of $\phi_{k}$,

$$
\begin{aligned}
i_{!}^{\prime}(1) & =c_{1}\left(K_{\mathbb{C}}{ }^{k} \otimes Q\right)=k c_{1}\left(K_{\mathbb{C}}\right)+c_{1}(Q) \\
& =k c_{1}\left(K_{\mathbb{C}}\right)+c_{1}-c_{1}\left(K_{\mathbb{C}}\right)=(k-1) c_{1}\left(K_{\mathbb{C}}\right)+c_{1} .
\end{aligned}
$$


Hence

$$
\begin{aligned}
j_{!} i_{k !}(1) & =j_{!} i_{k-1 !} i_{!}^{\prime}(1)=j_{!} i_{k-1 !}\left((k-1) c_{1}\left(K_{\mathbb{C}}\right)+c_{1}\right) \\
& =\left((k-1) c_{1}(\xi)+c_{1}\right) j_{!} i_{k-1 !}(1) \\
& =\left((k-1) c_{1}(\xi)+c_{1}\right)\left((k-2) c_{1}(\xi)+c_{1}\right) j_{!} i_{k-2 !}(1) \\
& =\left((k-1) c_{1}(\xi)+c_{1}\right) \ldots\left(c_{1}(\xi)+c_{1}\right) j_{!}(1) \\
& =\left(\sum_{t=0}^{k-1} \sigma_{t} c_{1}^{k-t-1} c_{1}(\bar{\xi})^{t}\right) j_{!}(1)
\end{aligned}
$$

where $\sigma_{t}$ is the parity of the value of the $t$ th symmetric function of $(k-1)$-variables evalutated on the integers $1, \ldots, k-1$. It turns out that $\sigma_{t} \equiv\left(\begin{array}{c}{[k / 2]} \\ t\end{array}\right)$ modulo 2. By using (4.4) we have

$$
\left[\Sigma_{1^{k}}(f)\right]^{c}=\pi_{!} j_{!} i_{k !}(1)=c_{1}^{k}+\sigma_{1} c_{2} c_{1}^{k-2}+\sigma_{2} c_{3} c_{1}^{k-3}+\ldots+\sigma_{k-1} c_{k} .
$$

In particular,

$$
\left[\Sigma_{1^{k}}(f)\right]^{c} \equiv f^{*} c_{1}(T M)^{k} \quad \text { modulo 2-torsion elements. }
$$

In the case of unoriented $L$, we use (co)homology with $\mathbb{Z}_{2}$-coefficients, and the formula (4.9) remains valid after changing $c_{i}$ to $w_{2 i}\left(:=w_{2 i}\left(T M^{\prime}-T L \oplus T L\right)\right)$, see Remark (4.7). Thus, for example,

$$
\left[\Sigma_{1}(f)\right]_{2}^{c}=w_{2}, \quad\left[\Sigma_{1,1}(f)\right]_{2}^{c}=w_{2}^{2}+w_{4}, \quad\left[\Sigma_{1,1,1}(f)\right]_{2}^{c}=w_{2}^{3}+w_{2} w_{4}, \quad \text { etc. }
$$

This completes the proof of Theorem (1.2).

Finally, we note that by the form of $(4.9),\left[\Sigma_{1^{k}}(f)\right]^{c} \in H^{2 k}(L ; \mathbb{Z})$ depends only on the homotopy type of $f$, so this completes the proof of Corollary (1.4).

5. Local Maslov indices and characteristic numbers. In this section, we deal with the case of $n=2$, and redefine the local Maslov index according to [I-O]. Let $f$ be an isotropic mapping from a closed surface $S$ to a symplectic 4-manifold $M$ such that the singularities of $f$ are all isolated.

Fix an almost complex structure of $T M$ compatible with $\omega$, and consider the section $\phi_{1}:=\rho(d f): S \rightarrow \operatorname{Hom}_{\mathbb{C}}\left(T S_{\mathbb{C}}, T M^{\prime}\right)$. For any point $p \in S$, choose a local trivialization of $\operatorname{Hom}_{\mathbb{C}}\left(T S_{\mathbb{C}}, T M^{\prime}\right)$ over a small disk $D$ in $S$ centered $p$ such that $\phi_{1}$ is non-singular over $D-p$. Then $\phi_{1}$ induces a map $D-p \rightarrow G L(2, \mathbb{C})$. When we take a local orientation of $D$ (for oriented $S$, we take the compatible one), the local Maslov index of $f$ at $p$, denoted by $m(f, p)$, is defined to be the homological degree of the composite map $D-p \rightarrow$ $G L(2, \mathbb{C}) \rightarrow \mathbb{C}-\{0\}$ where the second arrow is defined by taking the determinant. It is easy to see that this index $m(f, p)$ is independent of the choice of the compatible complex structure and the local trivialization. We note that $m(f, p)$ is half the value originally defined by Givental', since he used the square of the determinant. We let $m(f, p)_{2}$ denote the parity of $m(f, p)$, which is independent of the choice of the local orientation.

Now Corollary (1.5) is easily proved as follows. Taking a small perturbation of $\phi_{1}$ to be transverse to $\Sigma_{1}\left(T S_{\mathbb{C}}, T M^{\prime}\right)$ as in Remark (2.4), by the above definition of local Maslov indices, the sum of indices over all singular points of $f$ breaks into the transverse index 
of the perturbed section and $\Sigma_{1}\left(T S_{\mathbb{C}}, T M^{\prime}\right)$. This number is the same as the the Chern number $c=\left\langle c_{1}\left(T M^{\prime}\right),[S]\right\rangle$ by using the formula $(4.5)$ and the fact that $c_{1}\left(T L_{\mathbb{C}}\right)=0$. For the unoriented case, we note that $w_{2}(T S)=w_{1}(T S)^{2}$ (by Wu's formula, cf. [MS]), and hence $w_{2}\left(T M^{\prime}-T S \oplus T S\right)=w_{2}(T S)+w_{2}\left(T M^{\prime}\right)$. Thus the sum of $m(f, p)_{2}$ coincides with the characteristic number $\chi+w$. This completes the proof.

\section{References}

[A] V. I. Arnol'd, Singularities of Caustics and Wave Fronts, Kluwer Acad. Publ., 1990.

[AVGL] V. I. Arnol'd, V. A. Vasil'ev, V. V. Goryunov and O. P. Lyashko, Dynamical Systems VI, Singularity Theory I, Encyclopedia Math. Sci. 6, Springer, 1993.

[Au] M. Audin, Quelques remarques sur les surfaces lagrangiennes de Givental, J. Geom. Phys. 7 (1990), 583-598.

[G1] A. B. Givental', Lagrange imbeddings of surfaces and unfolded Whitney umbrella, Funktsional. Anal. Prilozhen. 20 (3) (1986), 35-41 (in Russian); English transl.: Funct. Anal. Appl. 20 (1986), 197-203.

[G2] - Singular Lagrange varieties and their Lagrangian mappings, Itogi Nauki Tekh., Sovrem. Probl. Mat. 33, VINITI, Moscow, 1988 (in Russian); English transl.: J. Soviet Math. 52 (1990).

[GG] M. Golubitsky and V. Guillemin, Stable Mappings and Their Singularities, Grad. Texts in Math. 14, Springer, Berlin, 1973.

[I1] G. Ishikawa, The local model of an isotropic map-germ arising from one-dimensional symplectic reduction, Math. Proc. Cambridge Philos. Soc. 111 (1992), 103-112.

[I2] - Maslov class of an isotropic map-germ arising from one dimensional symplectic reduction, in: Recent Developments in Differential Geometry, Adv. Stud. Pure Math. 22, Kinokuniya, 1993, 53-68.

[IO] G. Ishikawa and T. Ohmoto, Local invariants of singular surfaces in an almost complex four-manifold, Ann. Global Anal. Geom. 11 (1993), 125-133.

[K] L. Kleiman, The enumerative geometry of singularities, in: Real and Complex Singularities, Sijthoff and Noordhoff, 1977, 297-396.

[MS] J. Milnor and J. Stasheff, Characteristic Classes, Princeton Univ. Press, Princeton, N.J., and Univ. of Tokyo Press, Tokyo, 1974.

[P] I. R. Porteous, Simple singularities, in: Lecture Notes in Math. 192, Springer 1972, 286-307.

[R] F. Ronga, Le calcul des classes duals et singularités de Boardman d'ordre deux, Comment. Math. Helv. 47 (1972), 15-35.

[T] R. Thom, Les singularités des applications différentiables, Ann. Inst. Fourier (Grenoble) 6 (1955-56), 43-87.

[W] A. Weinstein, Lectures on Symplectic Manifolds, Regional Conf. Ser. in Math. 29, Amer. Math. Soc., 1977.

[Z] V. M. Zakalyukin, Generating ideals of singular Lagrange varieties, in: Theory of Singularities and its Applications, V. I. Arnol'd (ed.), Adv. Soviet Math. 1 (1990), 201-210. 